\title{
Fiber optic thermal health monitoring of composites
}

\author{
Meng-Chou Wu*, William P. Winfree and Jason P. Moore \\ NASA, Langley Research Center, MS 231, Hampton, VA, USA 23681-2199
}

\begin{abstract}
A recently developed technique is presented for thermographic detection of flaws in composite materials by performing temperature measurements with fiber optic Bragg gratings. Individual optical fibers with multiple Bragg gratings employed as surface temperature sensors were bonded to the surfaces of composites with subsurface defects. The investigated structures included a 10-ply composite specimen with subsurface delaminations of various sizes and depths. Both during and following the application of a thermal heat flux to the surface, the individual Bragg grating sensors measured the temporal and spatial temperature variations. The data obtained from grating sensors were analyzed with thermal modeling techniques of conventional thermography to reveal particular characteristics of the interested areas. Results were compared with the calculations using numerical simulation techniques. Methods and limitations for performing in-situ structural health monitoring are discussed.
\end{abstract}

Keywords: fiber Bragg grating, temperature sensor, thermal health monitoring, thermography, composite, delamination

\section{INTRODUCTION}

Fiber optic sensors have been extensively studied and proposed for temperature and strain sensing in heath monitoring systems of aerospace structures and materials as well as many other applications ${ }^{1-3}$ Compared to other sensors, Fiber optic sensors have the advantages of being lightweight and flexible, and requiring simpler wiring especially for distributed sensing. For an extensive heath monitoring system distributed sensors are an important requirement. Most efforts have focused on using distributed fiber optic strain sensing systems, with limited consideration given to temperature sensing systems. For fiber optic temperature sensing, the conventional techniques using Raman scattering can detect the average temperature over a long distance within a single fiber. However the spatial resolutions for temperature readings-averaging over a length of fiber-are typically about one meter. Therefore they are not suitable for measuring the thermal responses at specific locations of the investigated materials.

Another more attractive alternative is using fiber Bragg gratings (FBGs) for distributed temperature sensing (DTS). FBGs written in an optical fiber have a physical length of only a few millimeters. This size is nearly a point sensor. However, if multiple FBGs are written at different (multiplexing) wavelengths and read with a time domain demodulation system, the total number of these FBGs in a singe fiber is limited due to the finite bandwidth of the laser in the detector system. To solve the above problems of spatial resolution and limited sensors, recently a new technique for fiber optic DTS has been developed using low reflectivity FBGs. Under this technique low reflectivity FBGs were written at the same nominal wavelength and read with a frequency domain demodulation system. ${ }^{4,5}$ This technique allows a single fiber to contain hundreds of FBGs, employed as temperature and strain sensors.

An effective fiber optic DTS system has some advantages compared to a distributed strain sensing system for structure health monitoring. For a strain sensing system, in general, it is required to mechanically excite (or stress) the investigated structure or material. ${ }^{6}$ For some cases this is impractical, if not impossible. Obviously, using a DTS system requires the investigated material to be thermally excited. However, the temperature change of the material from the required thermal excitation can be only a few degrees or no more than tens of degrees. Such temperature variation is comparable with the temperature changes excited for conventional thermography techniques.

Conventional thermographic techniques, in general, utilize a flash or quartz lamp as a heating source and an infrared imager to detect the thermal response of the investigated material. The heating pulse duration typically ranges from a

*Meng-Chou.Wu-1@nasa.gov; phone 1757864 4951; fax 17578644914 
fraction of a second to a few seconds. The IR imager contains an array of several hundred by hundred detectors. These thermographic techniques are capable of large area inspection of aerospace structures and materials for their reliability and safety. One of the particularly important areas is the inspection of graphite fiber reinforced composite materials because they are being increasingly used as primary structures due to their high stiffness and strength to weight ratio. Of particular interest is the detection of delaminations that can appreciably reduce the compressive strength of a composite. Recently Winfree et al. successfully developed a physical model of two-layered systems for accurate reduction of the temporal thermal response of a composite with fabricated delaminations to the depths of the delaminations. ${ }^{7}$

In this paper we propose a new technique using a single optical fiber with multiple FBGs for the thermographic detection of flaws in materials and structures. The investigated structures included a 10-ply composite specimen with fabricated delaminations of various sizes and depths, similar to the one mentioned above. The optical fiber was bonded to the surface of the investigated composite. Both during and following the application of a thermal heat flux to the surface, the individual Bragg grating sensors measured the temporal and spatial temperature variations. The data obtained from individual FBGs were analyzed with thermal modeling to reveal particular characteristics within the area of interest.

\section{THEORY}

\subsection{FBG as a temperature sensor}

In general, a fiber Bragg grating can be characterized by its Bragg wavelength, which is the center wavelength of the light reflected from the grating. The Bragg wavelength is given as

$$
\lambda_{\mathrm{B}}=2 \mathrm{n}_{\mathrm{eff}} \Lambda,
$$

where $n_{\text {eff }}$ is the effective refractive index of the fiber core and $\Lambda$ the grating period. For a fiber Bragg grating bonded onto or embedded in a polymeric substrate, a change in the temperature causes a change in the grating period due to not only the thermal expansion of the fiber but also the strain induced by thermal expansion of the substrate. In addition, the refractive index of the fiber core changes because of the thermo-optic effect. Combining all the above effects, the shift in the Bragg wavelength due to a temperature change, $\Delta T$, is given as

$$
\delta \lambda_{\mathrm{B}} / \lambda_{\mathrm{B}}=\left(1-\mathrm{p}_{\mathrm{e}}\right) \delta l / l+\delta \mathrm{n}_{\mathrm{eff}} / \mathrm{n}_{\mathrm{eff}},
$$

where $\mathrm{p}_{\mathrm{e}}$ is the photoelastic constant of the optical fiber and $\delta l / l$ is the thermally induced strain of the fiber. In general the photoelastic constant, the fractional index change, and the thermal expansion coefficients of the fiber and the substrate are temperature dependent and nonlinear, especially at low temperatures. However, for a finite temperature change, especially for the temperatures around and above the room temperature, Eq. (2) can be rewritten as a linear form as, ${ }^{8}$

$$
\delta \lambda_{\mathrm{B}} / \lambda_{\mathrm{B}}=\left[\left(1-\mathrm{p}_{\mathrm{e}}\right) \alpha_{\mathrm{p}}+\xi\right] \Delta T,
$$

where $\xi=\left(1 / \mathrm{n}_{\mathrm{eff}}\right)\left(\hat{\partial} \mathrm{n}_{\mathrm{eff}} / \hat{\partial} T\right)$, is the thermo-optic coefficient of the fiber and $\alpha_{\mathrm{p}}$ is the thermal expansion coefficient of the substrate. Eq. (3) takes into account the conditions when the thermal expansion coefficient and the physical dimension of the substrate are much greater than those of the optical fiber. This simple equation allows the FBG to perform as a temperature sensor of the substrate.

\subsection{Thermal response of one and two-layered systems}

It is possible to derive a one-dimension series solution for a single layer with periodic heating using the method of images, if both sides of the layer are thermally insulated. The series solution for the surface where the periodic heating is applied is

$$
T_{f}(t)=\frac{F}{K} \sqrt{\frac{\kappa}{\omega}}\left(\cos \left(\omega t-\frac{\pi}{4}\right)+2 \sum_{n=1}^{\infty} e^{-n L \sqrt{\frac{2 \omega}{\kappa}}} \cos \left(\omega t-\frac{\pi}{4}-n L \sqrt{\frac{2 \omega}{\kappa}}\right)\right.
$$


where the periodic heat flux is given by $\mathrm{F} \cos (\omega t), K, \kappa$, and $L$ are the thermal conductivity, diffusivity and thickness of the layer. There are two extremes of interest. First, for the case of a very thick layer or $L>>(\kappa / 2 / \omega)^{1 / 2}$, the thermal response lags behind the periodic flux by $\pi / 4$. Secondly, for a very thin layer or $L<<(\kappa / 2 / \omega)^{1 / 2}$, the thermal response lags behind excitation by $\pi / 2$. All other thicknesses have phase differences that fall between these two limits.

Real delaminations in composites are air gaps between two layers in the composite. It is well known that for long times, the response of the temperature over the delamination is not characteristic of a single layer response. The typical explanation for this effect is that lateral heat flow around the delamination diffuses the heat to the backside of the delamination. However if the thickness of the air gap is such that the heat flow across the gap is much larger than the heat flow around the delamination, the gap's thermal resistance dominates the time response of the delamination. Note that if the thermal resistance of the delamination is sufficiently small, the time response at the front surface may not be significantly different than a single layer response.

A simple analytic solution does not exist for the one-dimensional heat flow in a multilayered material. A solution does however exist in Laplace space for two layers of thickness $l_{1}$ and $l_{2}$ coupled by an intermediate contact resistance $(R)$. Since the configuration of interest is a composite with a delamination, the first and second layers are assumed to have the same thermal conductivity $(K)$ and diffusivity $(\kappa)$. For the surface with the incident heating, the Laplace transform of the temperature impulse response is given by ${ }^{7}$

$$
T_{f}^{i}(p)=\frac{\left(\sinh \left(l_{1} q\right) \sinh \left(l_{2} q\right)+\cosh \left(l_{1} q\right)\left(\cosh \left(l_{2} q\right)+K q R \sinh \left(l_{2} q\right)\right)\right)}{K q\left(\sinh \left(l_{1} q\right) \cosh \left(l_{2} q\right)+\left(\cosh \left(l_{1} q\right)+K q R \sinh \left(l_{1} q\right)\right) \sinh \left(l_{2} q\right)\right)}
$$

where $\mathrm{q}$ is $\sqrt{p / \kappa}$ and $p$ is the coordinate in Laplace space.

The flux input from the periodically cycled heat lamps is assumed to be

$$
f(t)=f_{0}(1-\cos (\omega t)) \text {, }
$$

where $f_{0}$ is the energy per area from the heating source and $t_{0}$ is the heating duration. While it is possible to include this into Eq. (5), the numerical inverse Laplace transform of the combined equation is found to be unstable for periodic heat

fluxes at times greater than a 3 or 4 periods. Therefore to determine the thermal response for the periodic heat source, the inverse Laplace transform Eq. (5) is found numerically and this response is convolved with Eq. (6),

$$
T_{f}(t)=\int_{0}^{t} T^{i}(t-\tau)(1-\cos (\omega \tau)) d \tau
$$

where $T^{i}(t)$ is the inverse of Eq. (4).

\section{EXPERIMENT}

The low (smaller than a few tenths of one percent) reflectivity FBGs used in this research were written in situ, into the optical fiber drawn using the NASA Langley optical fiber draw tower. They were written with a pulsed KrF-excimer laser of $248 \mathrm{~nm}$ and a Talbot interferometer arrangement mounted on the tower. The interferometer consisted of a phase mask functioning as a beam splitter and a pair of mirrors used to recombine the split beams to form an interference pattern. An aperture was placed in the laser beam path to control the grating length and spatial profile. ${ }^{9}$ FBG lengths for the present study were nominally $5 \mathrm{~mm}$. The grating pitch written into the fiber could be adjusted by changing the relative angle of the two mirrors. These single-mode fibers were drawn from commercially available germanium-doped preforms of high numerical apertures. The drawn fibers with FBGs were coated with polyimide to thicknesses ranging from 11 to 16 micrometers, then ink-marked to show grating locations. 
These low reflectivity FBGs were interrogated using a frequency domain demodulation system shown in Fig. 1. In this system, the fiber coupler $C_{1}$ and a pair of Faraday rotation mirrors (FRMs) form an in-fiber interferometer with an optical path difference of $2 n_{\text {eff }} L_{0}$, where $n_{\text {eff }}$ is the effective refractive index of the fiber core and $\mathrm{L}_{0}$ the length of the reference cavity. The signals are driven by the tuning of the laser and detected at the photo-detector $\mathrm{D}_{1}$. They are used to trigger the sampling of signal at $\mathrm{D}_{2}$, which is the output of another in-fiber interferometer formed with the fiber coupler $\mathrm{C}_{2}$, a broadband reflector, and a particular fiber Bragg grating at a distance of $\mathrm{L}_{\mathrm{i}}$. If there is a series of low reflectivity Bragg gratings written at the same wavelength on a single fiber at different locations, the reflected signals from each grating are superimposed and detected at $\mathrm{D}_{2}$. The detected signals are further processed (fast-Fouriertransformed) to obtain the spatial spectrum of all gratings, which displays the physical profiles of the gratings at different locations. The spatial spectrum of a particular grating can then be windowed for investigating an individual grating. Fig. 2 shows the spatial spectrum of two FBGs in a fiber with multiple gratings. The gratings have a physical length of about $5 \mathrm{~mm}$ and a separation distance of $10 \mathrm{~cm}$. The signals from the demodulation system are further processed for the strain (thermally induced strain, in this case) of each point within the physical length of a grating. Averaging over a certain number of points within the grating length is taken for the strain of the particular grating.

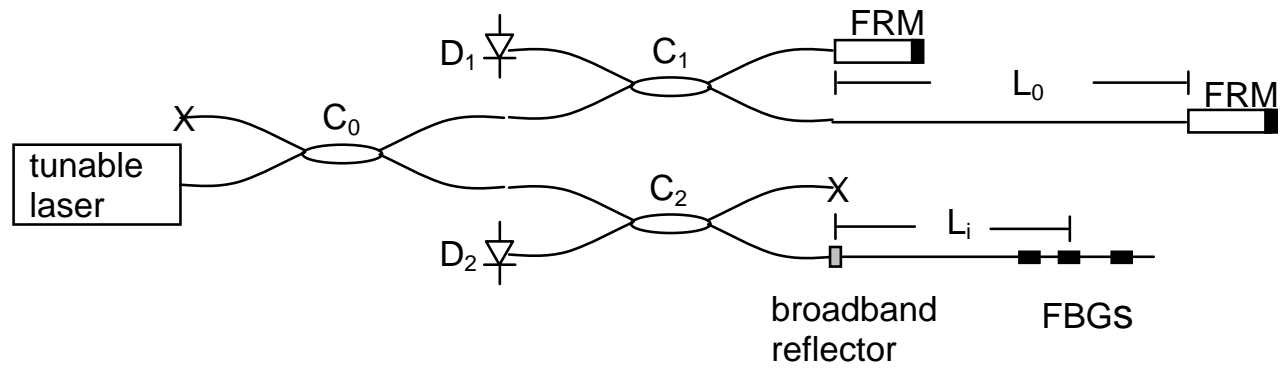

Fig. 1. Schematic diagram of a frequency domain demodulation system. Items $C_{0}, C_{1}$, and $C_{2}$ are fiber couplers. " $X$ " indicates that the unused port is terminated. Items $\mathrm{D}_{1}$ and $\mathrm{D}_{2}$ are detectors and FRMs are Faraday rotation mirrors.

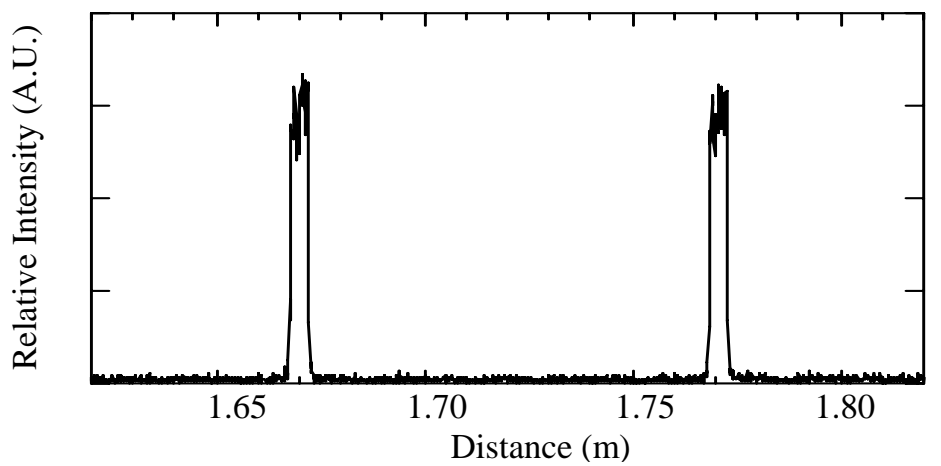

Fig. 2. The spatial spectrum of two FBGs with a physical length of about $5 \mathrm{~mm}$ and a separation distance of $10 \mathrm{~cm}$.

In this study, an optical fiber with 32 FBGs was bonded to an investigated composite specimen with Kapton ${ }^{\circledR}$ tape, as shown in Fig 3 (a). This bonding technique allowed the optical fiber to be bonded and taken off the sample quickly and 
neatly. At the same time it also allowed the fiber to sense the thermal strain for a temperature change up to $50^{\circ} \mathrm{C}$ without imminent slipping.

The specimen used for testing the viability of this measurement technique was a composite panel with 20 delaminations at specified depths. The 10 ply quasi-isotropic composite panel with a lay-up of [0,45,90,-45,0/,45,90,-45/,0,90/] was $31.75 \times 31.75$ centimeters and 0.19 centimeters thick (Fig. 3(a)). The delamination defect areas were squares with sizes of 3.8x3.8, 2.5x2.5, 1.9x1.9, and 1.3x1.3 square centimeters. The defects were buried at depths of 10, 20, 30, 40, and 50 percent of the total thickness. A schematic of the defect layout and FBG sensor numbers is shown in Fig. 3 (b). The optical fiber bonded to the specimen had FBGs with a separation (center-to-center) distance of 5.08 centimeters, which was the same as the separation distance of the squares in the same row. One FBG was placed at the center of each square. Five FBGs numbered 14-19 were outside the squares for comparison.

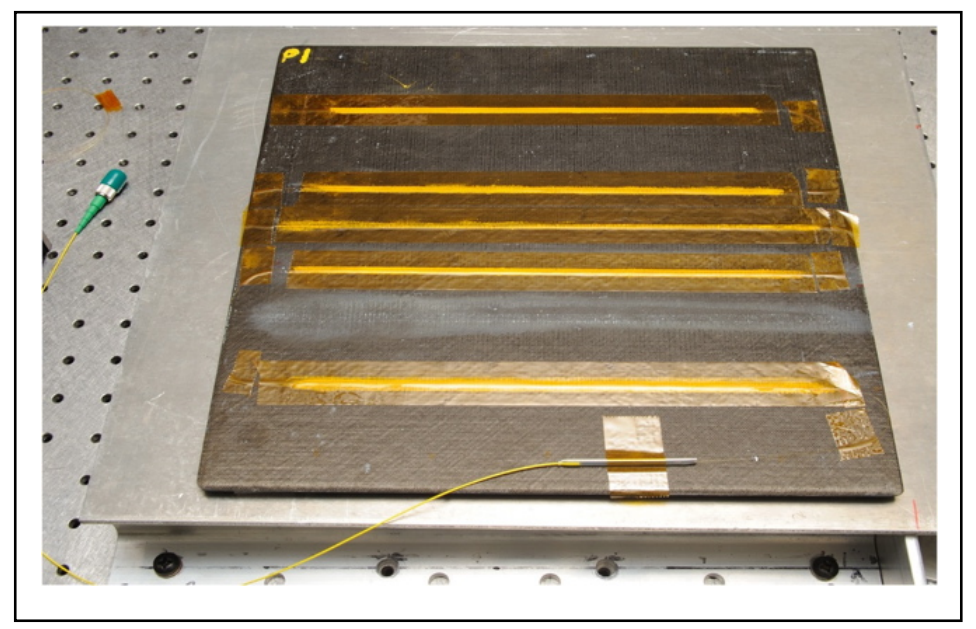

(a)

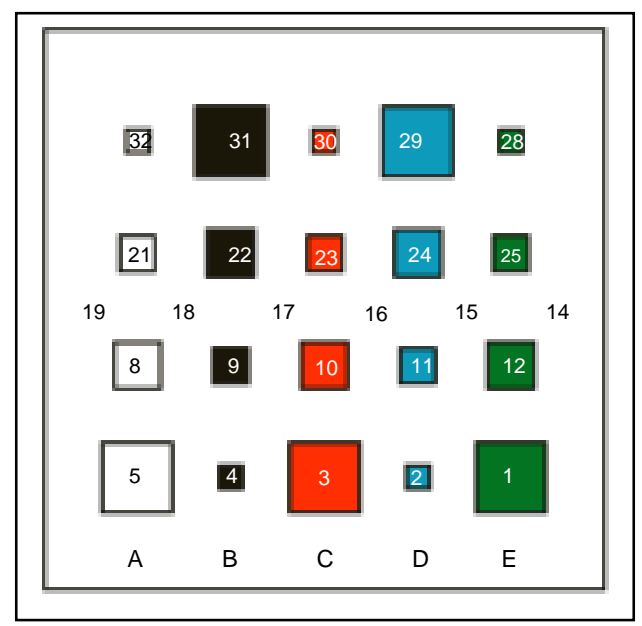

(b)

Fig. 3. (a) A single optical fiber with multiple Bragg grating sensors bonded onto the surface of a 10-ply-composite. (b) The fabricated delaminations of various sizes arranged in columns between plies 1-2 (A), 2-3 (B), 3-4 (C), 4-5 (D), 5-6 (E).

A quartz lamp was used to heat the front and the back surface of the specimen with a single or multiple heating cycles. The cyclic heating had typically a 2 second period with a $50 \%$ duty-cycle, and a total of about 20 second duration. The grating data acquisition was performed during and following the application of the heat flux to the surface at a rapidity rate of $100 \mathrm{~Hz}$. An IR camera was also used to record the temperatures of the surface. The experimental setup is shown in Fig. 4.

\section{RESULTS AND DISCUSSION}

Ideally, it is desirable to acquired data from all of the fiber optic sensors at one time. At the higher acquisition rate used for these measurements $(100 \mathrm{~Hz})$ this was not possible. Instead it was only possible to collect data along 5 adjacent gratings. For this reason the subsequent discussion will be limited to data acquired on gratings 8 to 12 . These gratings are over delaminations at 5 different depths in the composite, with the depth of the delamination increasing monotonically from gratings 8 to 12 . The time responses during a 1/2.048 Hz heating cycling of the composites as measured by gratings 8 to 12 are shown in Fig. 5 . As can be seen from this figure, there is no clear trend in the amplitude of the thermal responses. This is in part a result of the gratings not being calibrated to enable an absolute temperature measurement. 


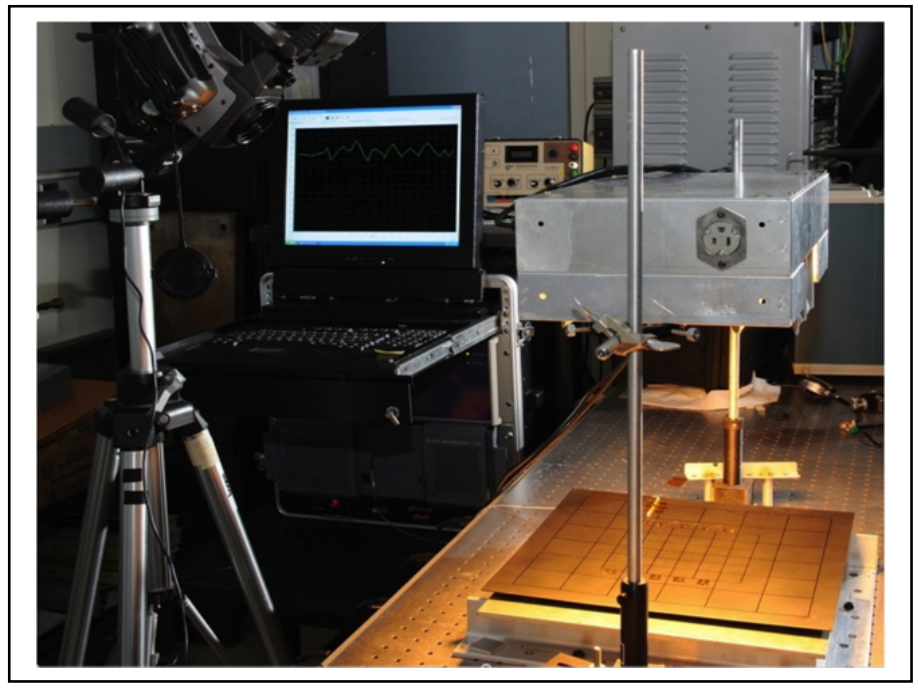

Fig. 4. The experimental setup of the fiber optic thermal health monitoring system with a quartz lamp as the heating source and a conventional IR camera.

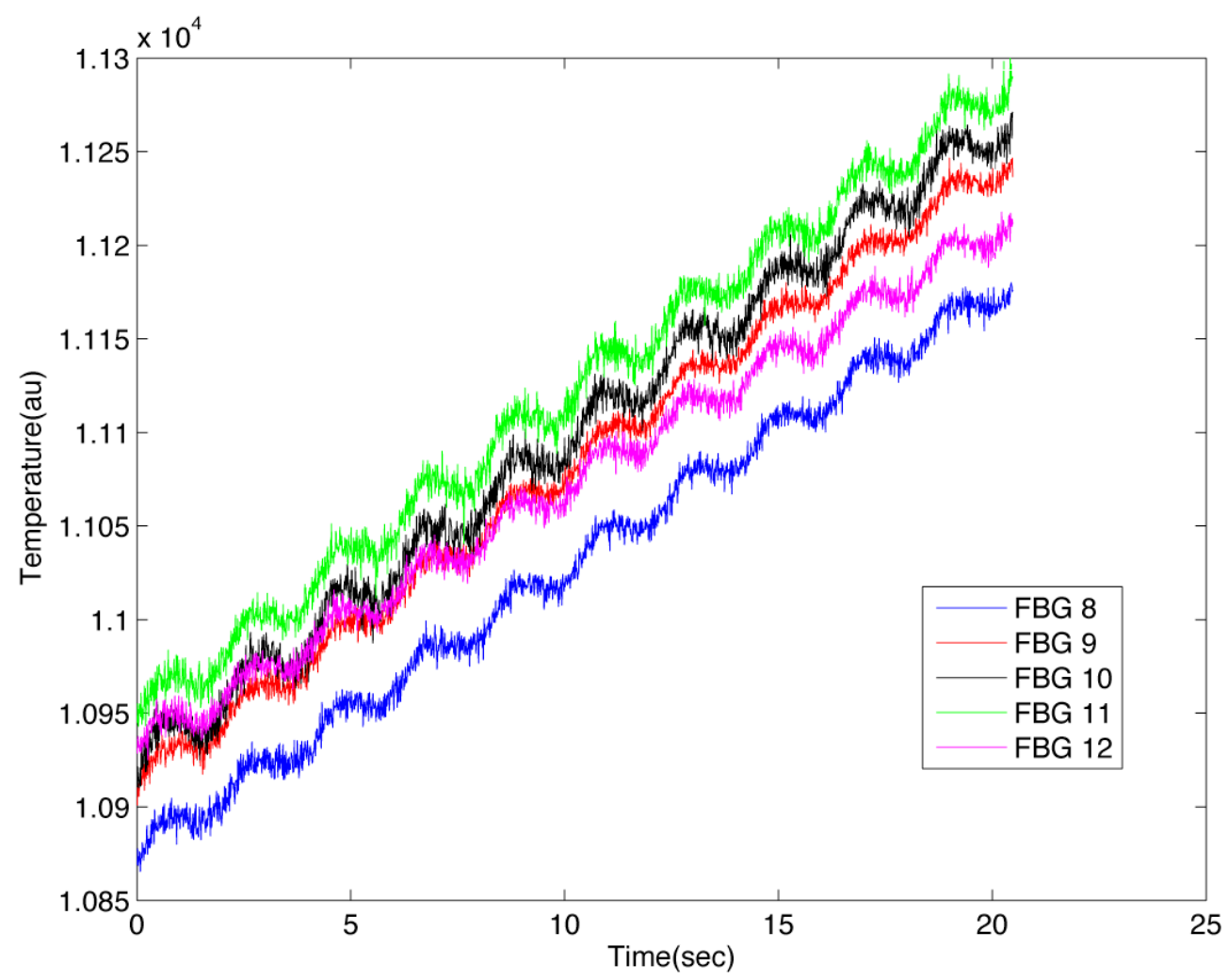

Fig. 5. The temperature measurements for 5 different gratings on the composite specimen as result of cycling a quartz lamp with a $1 / 2.048 \mathrm{~Hz}$ square wave. Each grating is over a different depth delamination in the composite. 


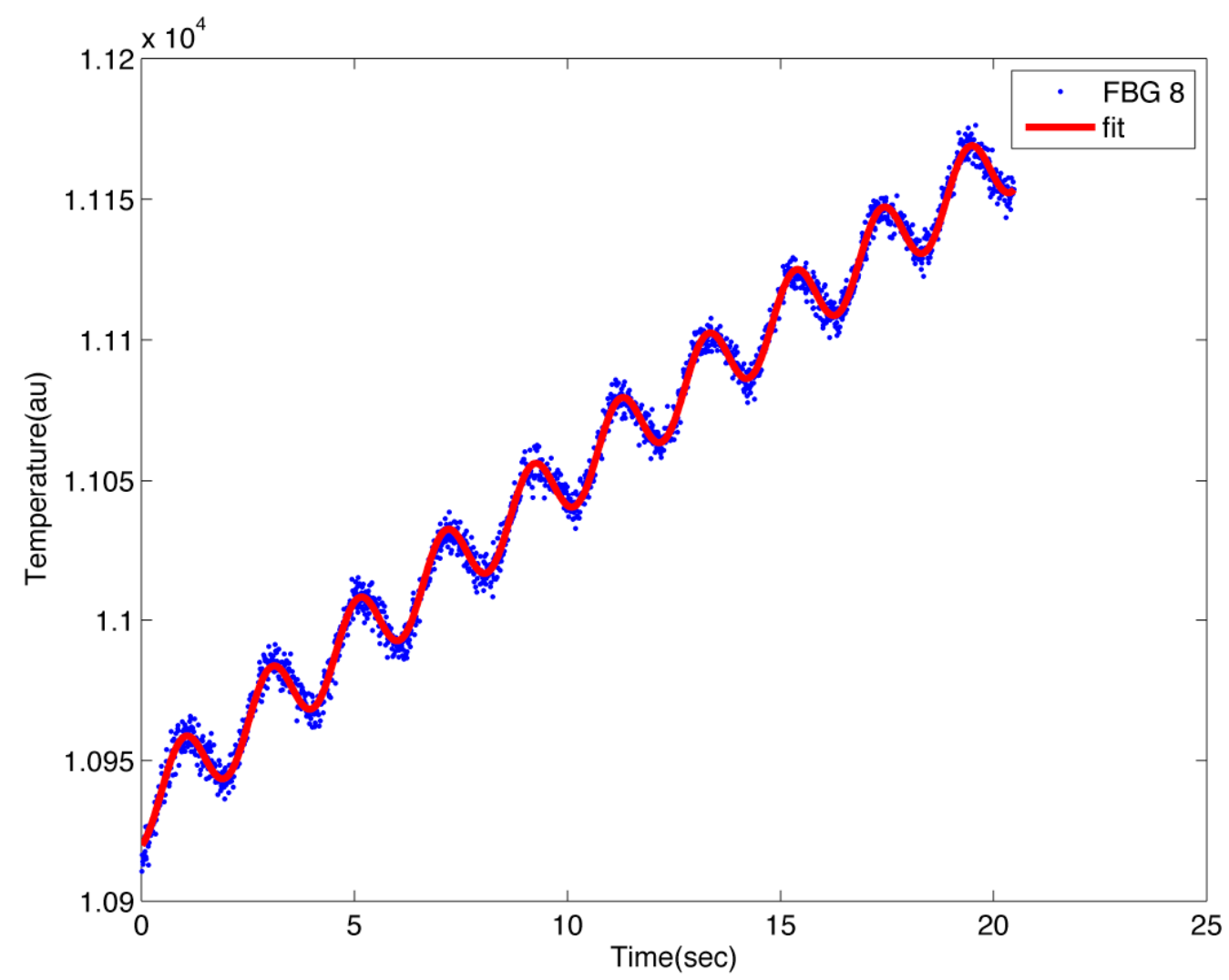

Fig 6. Linear least squares fit of the thermal response of the composite measured at FBG 8.

In section 2.2, it was noted that the phase of the response was a function of the thickness and thermal diffusivity of a layer and the frequency of the excitation; therefore it is of interest to find the phase of the response for each of the gratings. The phase angle has an additional advantage, since it is not dependent on the temperature amplitude, different gratings can be compared without calibration ${ }^{10}$. The cycling of power to the lamps does not result in a negative heat flux required for a purely cyclic heating of the specimen. Rather, a cyclic heating is superimposed on a static term. The static term results from an increase in the temperature of the panel during the test that needs to be accounted for to accurately determine the phase. To simultaneously account for the increase in temperature of the panel and calculate the phase, the data is fit with

$$
T(t)=\mathrm{A}_{1}+\mathrm{A}_{2} t+\mathrm{A}_{3} t^{2}+\mathrm{A}_{4} \cos (\omega t)+\mathrm{A}_{5} \sin (\omega t)
$$

where $\omega$ is the known angular frequency of the excitation $2 \pi / 2.048 \mathrm{~Hz}$. A typical fit of the measured temperature is shown in figure 6 . The phase angle is calculated from the $\arctan \left(A_{5} / A_{4}\right)$. The measured phase angle for the different depth of delaminations is shown in Fig. 7. As can be seen from the figure, there is a large phase angle for delaminations closer to the surface.

For comparison with the measured phase angles, the single layer solution (Eq. (4)), the convolved thermal response (Eq. (7)) and a three-dimensional finite element routine were used to calculate the phase angle for different depth delamination. The cyclic heating was not synchronized with the data acquisition; therefore the average phase shift for the different comparisons was set equal to the measured values. Common literature values of thermal properties of the composite were used. The thermal conductivity perpendicular to the fibers was assumed to be $0.97 \mathrm{~W} \mathrm{~m}^{-1} \mathrm{~K}^{-1}$ and 4.85 $\mathrm{W} \mathrm{m}{ }^{-1} \mathrm{~K}^{-1}$ along the fiber. The specific heat and density were assumed to be $1270 \mathrm{~J} \mathrm{~kg}^{-1} \mathrm{~K}^{-1}$ and $1490 \mathrm{~kg} \mathrm{~m}^{-1}$ respectively. A heat transfer coefficient of $8.063 \mathrm{~W} \mathrm{~m}^{-2} \mathrm{~K}^{-1}$ for convection loss at the front and back surface was used, based on the 
exponential decay in temperature at later times as measured by a fiber optic sensor at the center of the panel. For delaminations, $1.2 \mathrm{~cm}$ wide squared delaminations were placed at depths that corresponded to the interface between plies at positions corresponding to the squares in the schematic of the composite shown in Fig. 3(b). The smaller delaminations have the largest change in phase relative to the one-dimensional model due to in-plane heat flow.

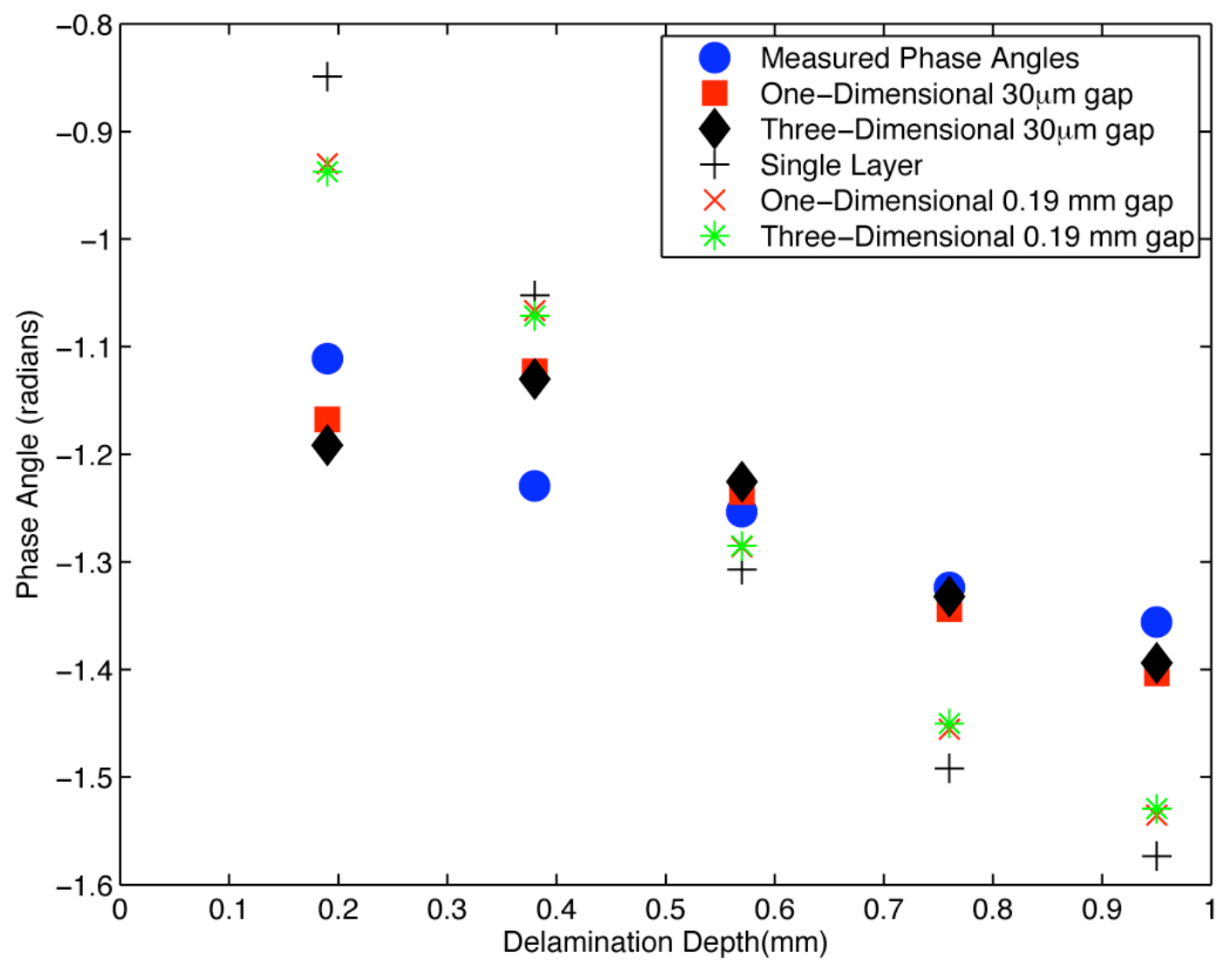

Fig 7. Phase measurement for different depths of measurements. The measured phase angles are compared to different model results from single-layer soultion, one-dimensional two-layer model, and three-dimensional finite element model.

The comparison of the model results to the measured results is shown in Fig. 7. As can be seen from the figure, the phase depends on the contact resistance (gap thickness/thermal conductivity of air) as well as the depth of the delamination. The best agreement between models and the measured phase angles is found assuming the air gap in the delamination to be $30 \mu \mathrm{m}$. Both the one-dimensional model (Eq. (7)) and the three-dimensional finite element model are in reasonably good agreement when assuming a $30 \mu \mathrm{m}$ air gap. This suggests there is relatively little in-plane heat flow in this time frame.

The single layer model shows the same trend as the measured phase angles, however it tends to overestimate the effect of depth on the phase angle. This appears to be a result of the finite contact resistance of the delamination significantly changing the phase angle. This is in agreement with both the one-dimensional and three-dimensional models for an air gap of $0.19 \mathrm{~mm}$, which is a relatively large contact resistance. The estimated phase angles for these models are in good agreement with each other and the single layer model. It is clear from these measurements, an estimation of the contract resistance is required for accurate depth measurements from the phase measurement. 


\section{CONCLUSION}

We have developed a new technique using distibuted FBG sensors for thermographic detection of flaws in materials and structures. Individual fibers with multiple FBGs employed as temperature sensors can be bonded to the surfaces of structures or embedded in the structures. By applying a cyclic thermal heat flux to the surface of the investigated structures, the individual Bragg grating sensors successfully measured the temporal and spatial temperature variations on the surface. These thermal responses were consistent with both one-dimensional and two-dimensional models of the thermal response for a delaminated composite. The measured phase shifts for delaminations of different depths are in good agreement with the model predictions assuming the delamination were $30 \mu \mathrm{m}$ air gaps. Future efforts will focus on developing the technique with a faster detection system, detecting more FBGs at one time, and assessing its potential for performing thermal health monitoring of aerospace structures and materials.

\section{REFERENCES}

1. C. R. Giles, “Lightwave applications of fiber Bragg gratings,” J. Lightwave Technol. 15, 1391-1404 (1997).

2. K. O. Hill, Y. Fujii, D. C. Johnson, and B. S. Kawasaki, "Photosensitivity in optical waveguides: application to reflection filter fabrication, “ Appl. Phys. Lett. 32, 647-649 (1978).

3. A. D. Kersey, M. A Davis, H. J. Patrick, M. LeBlanc, K. P. Koo, C. G. Askins, M.A. Putnam, E. J. Friebele, "Fiber grating sensors,” J. Lightwave Technol. 15, 1442-1463 (1997).

4. M. Froggatt and J. Moore, "Distributed measurement of static strain in an optical fiber with multiple Bragg gratings at nominally equal wavelengths,” Appl. Opt. 37, 1741-1746 (1998).

5. M.-C. Wu, R. Pater, and S. L. DeHaven, "Effects of coating and diametric load on fiber Bragg gratings as cryogenic temperature sensors,” Proc. SPIE, 6933, 693303 (2008).

6. S. W. James, M. L. Dockney, and R. P. Tatam, "Simultaneous independent temperature and strain measurement using in-fiber Bragg grating sensors,” Electron. Lett. 32, 1133-1134 (1996).

7. W. P. Winfree and J. Zalameda, "Thermographic determination of delamination depth in composites," in Thermosense XXV, Proc. SPIE 5073, 363-373 (2003).

8. S. Gupta, T. Mizunami, T. Yamao, and T. Shimomura, "Fiber Bragg grating cryogenic temperature sensors," Appl. Opt. 25, 5202-5205 (1996).

9. M.-C. Wu and R. S. Rogowski, "Fabrication of self-apodized short-length fiber Bragg gratings," Appl. Opt. 42, 5017-5023 (2003).

10. X. Maldague, S. Marinetti, “Pulse Phase Infrared Thermography,” J. Appl. Phys, 79, 2694-2698 ,1996. 